phys. stat. sol. (b) 111, 631 (1982)

Subject classification: 6 and $13.4 ; 22$

Institut für Theoretische Physik und Physikalisches Institut der Universität Stuttgart ${ }^{1}$ )

\title{
The Influence of the Dynamic Jahn-Teller Effect on the Phonon-Scattering Mechanism at Acceptors in Semiconductors with Cubic Symmetry
}

By

\author{
E. Sigmund and K. Lassmann
}

Phonon scattering experiments of various types in cubic semiconductors doped with deep effective mass acceptors indicate an extra resonance scattering at some meV. It is shown, using Green's function and transformation techniques that these resonances are due to a dynamic Jahn-Teller effect of the $\Gamma_{\mathbf{8}}$ acceptor ground state.

Phononen-Streuexperimente verschiedener Art weisen in kubischen, mit tiefen EffektivmassenAkzeptoren dotierten Halbleitern eine zusätzliche Resonanzstreuung bei einigen $\mathrm{meV}$ auf. Es wird mit Hilfe von Greenschen Funktionen und Transformationsmethoden gezeigt, daß diese Resonanzen durch einen dynamischen Jahn-Teller-Effekt des $\Gamma_{\mathbf{8}}$-Akzeptorgrundzustandes hervorgerufen werden.

\section{Introduction}

The ground state of acceptors in cubic semiconductors is fourfold degenerate $\left(\Gamma_{8}\right)$ and the interaction with the lattice vibrations leads to the possibility of a Jahn-Teller effect [1]. The extended nature of the wave function of these defects [2] has two important consequences:

(i) the interaction is mainly with long-wavelength acoustic phonons,

(ii) because of the large elastic energy associated with the center and the small Jahn-Teller energy residual static strains may destroy the Jahn-Teller effect.

There is a series of phonon experiments [3 to 6] and optical measurements $[7,8]$ which indicate a resonance energy in the $\mathrm{meV}$ range for the deeper acceptors GaAs $(\mathrm{Mn}), \mathrm{GaP}(\mathrm{Zn}), \mathrm{Si}(\mathrm{In})$, and $\mathrm{Si}(\mathrm{B})$. These energies are much larger than the splitting due to random internal fields, which are of the order of 10 to $100 \mu \mathrm{eV}$ in these crystals. In the following we want to show that these resonance energies are related to the dynamic Jahn-Teller effect. The acceptor-hole-lattice interaction Hamiltonian can be written in the form

$$
H_{\mathrm{I}}=\sum_{q \lambda}\left\{D^{\varepsilon}\left(\hat{\varrho}_{1} r_{1}^{q \lambda}+\hat{\varrho}_{2} q_{2}^{q \lambda}\right)+D^{\tau} \hat{\varrho}_{3}\left(\hat{\sigma}_{1} s_{1}^{q \lambda}+\hat{\sigma}_{2} s_{2}^{q \lambda}+\hat{\sigma}_{3} s_{3}^{q \lambda}\right)\right\}\left(b_{q \lambda}+b_{q \lambda}^{+}\right) .
$$

We have disregarded the coupling term which is diagonal in the electronic operators. It gives only a shift of the center of gravity of the valence band edge and does not contribute to the Jahn-Teller effect.

The following abbreviations are introduced:

$$
r_{1}^{q \lambda}=\left(\frac{h \omega_{q \lambda}}{2 M c_{q \lambda}^{2}}\right)^{1 / 2} f(q) \frac{1}{3}\left[2 \hat{q}_{z} \boldsymbol{e}_{\lambda z}-\hat{q}_{x} \boldsymbol{e}_{\lambda x}-\hat{q}_{\nu} \boldsymbol{e}_{\lambda y}\right],
$$

\footnotetext{
1) Pfaffenwaldring 57, D-7000 Stuttgart, FRG.
} 


$$
\begin{aligned}
r_{2}^{q \lambda} & =\left(\frac{h \omega_{q \lambda}}{2 M c_{q \lambda}^{2}}\right)^{1 / 2} f(q) \frac{1}{\sqrt{3}}\left[\hat{q}_{x} \boldsymbol{e}_{\lambda x}-\hat{q}_{y} \boldsymbol{e}_{\lambda y}\right], \\
s_{1}^{q \lambda} & =\left(\frac{h \omega_{q \lambda}}{2 M c_{q \lambda}^{2}}\right)^{1 / 2} f(q) \frac{1}{\sqrt{3}}\left[\hat{q}_{z} \boldsymbol{e}_{\lambda y}+\hat{q}_{y} \boldsymbol{e}_{\lambda z}\right], \\
s_{2}^{q \lambda} & =\left(\frac{\hbar \omega_{q \lambda}}{2 M c_{q \lambda}^{2}}\right)^{1 / 2} f(q) \frac{1}{\sqrt{3}}\left[\hat{q}_{z} \boldsymbol{e}_{\lambda x}+\hat{q}_{x} \boldsymbol{e}_{\lambda z}\right], \\
s_{3}^{q \lambda} & =\left(\frac{h \omega_{q \lambda}}{2 M c_{q \lambda}^{2}}\right)^{1 / 2} f(q) \frac{1}{\sqrt{3}}\left[\hat{q}_{x} \boldsymbol{e}_{\lambda y}+\hat{q}_{y} \boldsymbol{e}_{\lambda x}\right] .
\end{aligned}
$$

Here the long-wavelength approximation is used, i.e. only acoustic phonons are coupled.

$b_{q \lambda}$ and $b_{q \lambda}^{+}$are the annihilation and creation operators for the phonon with wave vector $\boldsymbol{q}$ in the branch $\lambda . \omega_{q \lambda}$ is the angular frequency and $c_{q \lambda}$ the velocity of sound. $\hat{\boldsymbol{q}}$ is the unit vector along $\boldsymbol{q}$ and $\boldsymbol{e}_{q \lambda}$ is the polarization vector of the phonon. $M$ is the mass of the crystal. $D^{e}$ and $D^{\tau}$ are the deformation potential constants for $[1,0,0]$ and $[1,1,1]$ strains, respectively. These constants differ from the experimentally measured values of $D_{u}^{a}$ and $D_{\mathrm{u}^{\prime}}^{a}$ shown in Table 1 . The difference between the two sets

Table 1

Experimental and theoretical values of resonance energies

\begin{tabular}{lllll}
\hline & $\mathrm{GaAs}(\mathrm{Mn})$ & $\mathrm{GaP}(\mathrm{Zn})$ & $\mathrm{Si}(\mathrm{In})$ & $\mathrm{Si}(\mathrm{B})$ \\
\hline experimental values $(\mathrm{meV})$ & $3.1 \pm 0.3$ & $2.7 \pm 0.3$ & $4.2 \pm 0.2$ & $\left.2^{*}\right)$ \\
\hline theoretical values $(\mathrm{meV})$ & 2.6 & 2.3 & 4.0 & 1.9 \\
\hline
\end{tabular}

*) Value not quite sure from experiment.

of parameters lies in the Ham reduction of these coupling constants. The experimentally measured values are reduced by the Jahn-Teller interaction whereas for our calculation we need the unreduced values. In order to fit our experimental data we used as a first approximation the reduction factors calculated by Morgan [1] for the equal-coupling case. An extension of his numerical calculations to the case of unequalcoupling constants which is more realistic for our systems will be given in the next future.

The original Hamiltonian, the electronic part of which is usually written in terms of the angular momentum operators $[9,10]$, is transformed in such a way as to contain $\hat{\varrho}_{i}$ and $\hat{\sigma}_{j}(i, j=1,2,3)$, which are Dirac's $4 \times 4$ matrices $[11,12]$ with the commutation relations

$$
\left.\begin{array}{l}
{\left[\hat{\varrho}_{i}, \hat{\varrho}_{j}\right]=2 i \hat{\varrho}_{k} ; \quad\left[\hat{\sigma}_{i}, \hat{\sigma}_{j}\right]=2 i \hat{\sigma}_{k},} \\
{\left[\hat{\varrho}_{i}, \hat{\varrho}_{i}\right]=0 ; \quad\left[\hat{\sigma}_{i}, \hat{\sigma}_{i}\right]=0 ; \quad\left[\hat{\varrho}_{i}, \hat{\sigma}_{j}\right]=0 . .}
\end{array}\right\}
$$

The fact that the wave function is extended over many lattice sites is reflected in an approximate way by the cut-off function [13]

$$
f(q)=\left[1+\frac{1}{4} a^{* 2} q^{2}\right]^{-2},
$$

where $a^{*}$ is the effective Bohr radius. 


\section{Phonon Relaxation Rate}

To discuss the phonon scattering at these acceptor systems we have to calculate the scattering rate (or inverse lifetime) which for a single phonon $q, \lambda$ is given by [14]

$$
\tau_{q \lambda}^{-1}=-\omega_{q \lambda}^{-1} \lim _{\varepsilon \rightarrow 0} \operatorname{Im} T\left(\omega_{q \lambda}+\mathrm{i} \varepsilon\right)_{q \lambda, q \lambda} .
$$

$T\left(\omega_{q \lambda}+i \varepsilon\right)$ is the $T$-matrix defined by the phonon Green's functions $\left(G=G_{0}-G_{0} T G_{0}\right)$ of the unperturbed $\left(G_{0}\right)$ and perturbed $(G)$ crystals.

Following $[14,15]$, the phonon Green's functions can be replaced by Green's functions between the electronic operators $\hat{\varrho}_{i}$ and $\hat{\sigma}_{j}(i, j=1,2,3)$. Then (5) takes the form

$$
\tau_{q \lambda}^{-1}=4 \pi \lim _{\varepsilon \rightarrow 0} \operatorname{Im}\left\langle\left\langle\Lambda^{q \lambda} ; \Lambda^{q \lambda}\right\rangle,\right.
$$

where $A^{q \lambda}$ reads

$$
\Lambda^{q \lambda}=D^{\varepsilon}\left(\hat{\varrho}_{1} r_{1}^{q \lambda}+\hat{\varrho}_{2} r_{2}^{q \lambda}\right)+D^{\tau} \hat{\varrho}_{3}\left(\hat{\sigma}_{1} s_{1}^{q \lambda}+\hat{\sigma}_{2} s_{2}^{q \lambda}+\hat{\sigma}_{3} s_{3}^{q \lambda}\right) \text {. }
$$

We see that treating (6) 25 different Green's functions have to be calculated, 15 of which are independent of each other. The mean scattering rate for phonons of frequency $\omega_{q \lambda}=\omega$ is defined by

$$
\left\langle\tau(\omega)^{-1}\right\rangle=\sum_{q \lambda} \tau_{q \lambda}^{-1} \delta\left(\omega_{q \lambda}-\omega\right) /\left\{\sum_{q \lambda \lambda} \delta\left(\omega_{q \lambda}-\omega\right)\right\}
$$

In the following we consider a longitudinally polarized phonon in a $[1,0,0]$ direction. This simplifies the further treatments considerably, for in this case we are only concerned with the "spin-spin" Green's function $\left\langle\hat{\varrho}_{1} ; \hat{\varrho}_{1}\right\rangle$. Using the equation of motion method we expand the Green's function hierarchy up to the fourth order. In second order we factorize the quadratic forms of the phonon operators,

$$
\left\langle\left\langle\left(b_{q \lambda}+b_{q \lambda}^{+}\right)\left(b_{q^{\prime} \lambda^{\prime}}+b_{q^{\prime} \lambda^{\prime}}^{+}\right) \hat{\xi}_{i} ; \hat{\xi}_{j}\right\rangle\right\rangle=\left\langle\left(b_{q \lambda}+b_{q \lambda}^{+}\right)\left(b_{q^{\prime} \lambda^{\prime}}+b_{q^{\prime} \lambda^{\prime}}^{+}\right)\right\rangle_{0}\left\langle\left\langle\hat{\xi}_{i} ; \hat{\xi}_{j}\right\rangle\right\rangle \text {. }
$$

$\hat{\xi}_{i}, \hat{\xi}_{j}$ are operators belonging to the algebra built up by the operators $\hat{\varrho}_{i}$ and $\hat{\varrho}_{3} \hat{\sigma}_{i}$ $(i=1,2,3)$ of the initial Hamiltonian. In fourth order we close the hierarchy by taking into account only the inhomogeneous part of the Green's functions. To make the results more accurate we calculate the thermal expectation values not in the original but in a transformed space. This can be done by using an exponential transformation $U=\exp (S)$ of the form [16]

$$
S=\sum_{q \lambda} \omega_{q \lambda}^{-1} \Lambda^{q \lambda}\left(b_{q \lambda}-b_{q \lambda}^{+}\right) / \hbar
$$

The transformed Hamiltonian contains no longer linear terms in the electron-phonon coupling strength, since the relation

$$
\left[H_{0}, S\right]=-H_{\mathrm{I}}
$$

holds. ( $H_{0}$ is the diagonal Hamiltonian of the uncoupled systems). This leads to the energies and expectation values which are exact at least up to second order in the coupling parameters.

For example we get

$$
\begin{aligned}
& \left\langle\left(b_{q \lambda}-b_{q \lambda}^{+}\right)\left(b_{q^{\prime} \lambda^{\prime}}+b_{q^{\prime} \lambda^{\prime}}^{+}\right)\right\rangle_{0}=\delta_{q q^{\prime}} \delta_{\lambda \lambda^{\prime}}, \\
& \left\langle\left(b_{q \lambda}+b_{q \lambda}^{+}\right)\left(b_{q^{\prime} \lambda^{\prime}}+b_{q^{\prime} \lambda^{\prime}}^{+}\right)\right\rangle_{0}= \\
& =\delta_{q q^{\prime}} \delta_{\lambda \lambda^{\prime}} \operatorname{coth}\left(h \omega_{q \lambda} \beta / 2\right)+4\left\{\sum_{i=1}^{2} r_{i}^{q \lambda} r_{i}^{q^{\prime} \lambda^{\prime}}+\sum_{i=1}^{3} s_{i}^{q \lambda} \delta_{i}^{q^{\prime} \lambda^{\prime}}\right\} / h \omega_{q \lambda} h \omega_{q^{\prime} \lambda^{\prime}}, \\
& \left\langle\left(b_{q \lambda}+b_{q \lambda}^{+}\right) \hat{\varrho}_{1}\right\rangle_{0}=\left(-2 r_{1}^{q \lambda} / h \omega_{q \lambda}\right)+\ldots, \text { etc. }
\end{aligned}
$$

with $1 / \beta=k_{\mathrm{B}} T$ and $k_{\mathrm{B}}$ the Boltzmann constant. 
For the crystal an isotropic model is introduced and all sums are converted into integrals over frequency dependent and angular dependent parts, which can be solved analytically for zero temperature. As a final result for the mean scattering rate we get the Lorentzian-like form

$$
\left\langle\tau(\omega)^{-1}\right\rangle=\frac{P(\omega)}{\{\omega-\Delta(\omega)\}^{2}+\Gamma(\omega)^{2}} .
$$

The functions $P(\omega), \Delta(\omega)$, and $\Gamma(\omega)$ as calculated in the described way are exact up to the fourth order in the coupling constants.

They read for $T=0 \mathrm{~K}$

$$
\begin{aligned}
P(\omega)= & {[\{\omega-\Delta(\omega)\} \beta(\omega)-\alpha(\omega) \Gamma(\omega)] \gamma(\omega), } \\
\alpha(\omega)= & \frac{4 \omega}{\pi \hbar^{2}} \sum_{\lambda} \sum_{j \neq 1} \delta^{\lambda} R_{(2)}^{\lambda} A_{j}^{\lambda}, \\
\beta(\omega)= & \frac{2 \omega^{2}}{\hbar^{2}} \sum_{\lambda} \sum_{j \neq 1} \delta^{\lambda} f^{2}(\omega) A_{j}^{\lambda}, \\
\Delta(\omega)= & \frac{4 \omega}{h} \sum_{\lambda} \sum_{j \neq 1} \delta^{\lambda} R_{(1)}^{\lambda} A_{j}^{\lambda}+\frac{16 \omega}{h^{2}} \sum_{\lambda, \lambda^{\prime}} \sum_{j \neq 1} \delta^{\lambda} \delta^{\lambda^{\prime}} R_{(2)}^{\lambda} R_{(3)}^{\lambda^{\prime}} A_{j}^{\lambda^{\prime}} A_{j}^{\lambda^{\prime}}- \\
& -\frac{4}{h} \sum_{\lambda} \sum_{j \neq 1} \delta^{\lambda} R_{(4)}^{\lambda} A_{j}^{\lambda}, \\
\Gamma(\omega)= & \frac{8 \pi \omega^{2}}{h^{2}} \sum_{\lambda, \lambda^{\prime}} \sum_{j \neq 1} \delta^{\lambda} \delta^{\lambda^{\prime}} f^{2}(\omega) R_{(3)}^{\lambda^{\prime}} A_{j}^{\lambda} A_{j}^{\lambda^{\prime}}, \\
\gamma(\omega)= & \frac{4}{9}\left(D^{\varepsilon}\right)^{2} \frac{1}{\varrho_{M}} p \frac{\omega^{3} f^{2}(\omega)}{\bar{c}^{5} \pi s \varrho_{0}}, \\
X_{\lambda}(\omega)= & {[f(\omega)]^{1 / 2}=1 /\left(1+u_{\lambda}^{2}\right), }
\end{aligned}
$$

where

$$
\frac{1}{\bar{c}}=\left[\frac{1}{3}\left(\frac{1}{c_{1}^{3}}+\frac{2}{c_{\mathrm{t}}^{3}}\right)\right]^{1 / 3}, \quad \delta^{\lambda}=1 /\left(16 \pi^{3} \varrho_{M} c_{\lambda}^{5}\right), \quad u_{\lambda}=\left(a^{*} \omega / 2 c_{\lambda}\right),
$$

$\left(\varrho_{M}=M / V\right) . s$ counts the number of atoms in the unit cell, $p$ is the defect concentration, and the normalized density of phonon states reads

$$
o_{0}=\sum_{\lambda}\left(V / 2 \pi \pi^{2}\right)\left(\omega^{2} / c_{\lambda}^{3}\right)
$$

The functions

$$
\begin{aligned}
& R_{(1)}^{\lambda}=-\frac{2}{3}\left[\frac{c_{\lambda}}{a^{*}}\right]^{2}-\frac{1}{2} \omega^{2} X_{\lambda}(\omega)^{4}\left\{\frac{11}{6}+2 \ln u_{\lambda}+3 u_{\lambda}^{2}+\frac{3}{2} u_{\lambda}^{4}+\frac{1}{3} u_{\lambda}^{6}\right\}, \\
& R_{(2)}^{\lambda}=\frac{5}{16}\left[\frac{c_{\lambda}}{a^{*}}\right]+\frac{1}{2} u_{\lambda} \omega\left\{\frac{5}{16} X_{\lambda}(\omega)+\frac{3}{8} X_{\lambda}(\omega)^{2}+\frac{1}{2} X_{\lambda}(\omega)^{3}+X_{\lambda}(\omega)^{4}\right\}, \\
& R_{(3)}^{\lambda}=\frac{1}{4}\left[\frac{c_{\lambda}}{a^{*}}\right]^{3}, \\
& R_{(4)}^{\lambda}=-R_{(3)}^{\lambda}+\omega^{2} R_{(2)}^{\lambda}
\end{aligned}
$$


result from the $\omega_{q \lambda}$ dependent integrations, whereas the $A_{j}^{\lambda}$ are the solutions of the angular dependent integrations,

$$
\begin{aligned}
& A_{2}^{1}=\frac{16}{45} \pi\left(D^{s}\right)^{2}, \quad A_{2}^{2}=\frac{4}{45} \pi\left(D^{\varepsilon}\right)^{2}, \quad A_{2}^{3}=\frac{4}{9} \pi\left(D^{\varepsilon}\right)^{2}, \\
& A_{3}^{1}=A_{4}^{1}=A_{5}^{1}=\frac{16}{45} \pi\left(D^{\tau}\right)^{2}, \\
& A_{3}^{2}=A_{4}^{2}=\frac{14}{45} \pi\left(D^{\tau}\right)^{2}, \quad A_{5}^{2}=\frac{4}{45} \pi\left(D^{x}\right)^{2}, \\
& A_{3}^{3}=A_{4}^{3}=\frac{2}{9} \pi\left(D^{x}\right)^{2}, \quad A_{5}^{3}=\frac{4}{9} \pi\left(D^{x}\right)^{2},
\end{aligned}
$$

$\Gamma(\omega)$ represents the "width" of the resonance. For zero temperature it contains no quadratic terms in the coupling parameters. The quadratic terms, however, are important at finite temperatures.

In Fig. 1 the relaxation rate of a longitudinal phonon scattered by the JT defect In in $\mathrm{Si}$ as derived in this paper is plotted $\left(\tau_{\mathrm{J}^{\mathrm{T}}}^{-1}\right)$. For comparison the result of a secondorder perturbational calculation $\left(\tau_{\mathbf{P}}^{-1}\right)$ is given. The small width of our result is caused by the resonance structure of (13). For $\omega \rightarrow 0 \tau_{\mathrm{JT}}^{-1}$ approaches $\tau_{\mathrm{P}}^{-1}$ asymptotically.

\section{Resonance Frequencies}

In this paper we restrict ourselves to the discussion of the resonance frequency. It is given by the relation

$$
\omega-\Delta(\omega)=0 \text {. }
$$

We have calculated the resonance frequencies for several acceptors. The results are written in Table 1. In Table 2 the parameters are given which enter into the calculation. Since as yet we did not calculate the Ham reduction factors $p^{\varepsilon}$ and $p^{\tau}$ for the ratios $D^{\varepsilon} / D_{\mathrm{u}}^{\mathrm{a}}$ and $D^{\tau} / D_{\mathrm{u}}^{\mathrm{a}}$, respectively, we have taken them as one fit parameter $p^{\varepsilon}=$ $=p^{r}=p$, the range of its possible values is given by the equal coupling theory (Morgan [1]). It turned out that the Bohr radius $a^{*}$ had to be somewhat reduced as compared with the value resulting from the effective mass approximation.

The experimental data are found by the following measurements:

a) Thermal conductivity measurements for $\mathrm{Si}(\mathrm{In})$ and $\mathrm{GaAs}(\mathrm{Mn})$. These indicate a pronounced additional scattering mechanism at temperatures around 23 and $12 \mathrm{~K}$, respectively [3].

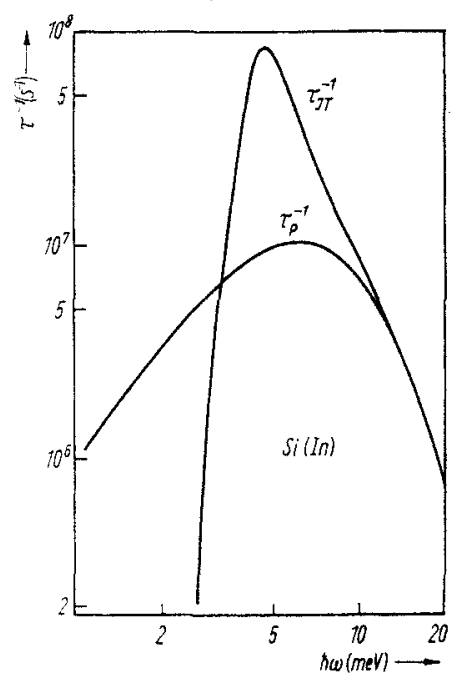

b) Measurements with quasimonochromatic phonons generated by superconducting tunneling junctions in $\mathrm{Si}(\mathrm{In})$. They show a broad extra scattering with maximum at $4.1 \mathrm{meV}[4]$.

c) Luminescence spectrum of bound excitons in $\mathrm{Si}(\mathrm{In})$. A broad satellite line occurs at about $4.1 \mathrm{meV}$ above the ground state [8].

Fig. 1. Scattering rate of our result $\left(\tau_{\mathcal{J}}^{-1}\right)$ and the perturbational result $\left(\tau_{\vec{p}}^{-1}\right)$ in dependence on the phonon energy. For the calculation we assumed an In concentration of $1 \times 10^{16} \mathrm{~cm}^{-3}$ 
Table 2

Experimental values of physical parameters used in the calculation (explanation see text)

\begin{tabular}{lllll}
\hline & $\mathrm{GaAs}(\mathrm{Mn})$ & $\mathrm{GaP}(\mathrm{Zn})$ & $\mathrm{Si}(\mathrm{In})$ & $\mathrm{Si}(\mathrm{B})$ \\
\hline$D_{\mathrm{u}}^{\mathrm{a}}(\mathrm{eV})$ & $0.53[17]$ & $1.56[7]$ & $1.47[18]$ & $2.13[18]$ \\
$D_{\mathbf{u}^{\prime}}^{\mathrm{a}}(\mathrm{eV})$ & 0.69 & 3.03 & 2.32 & 3.20 \\
$p$ & 0.5 & 0.8 & 1.0 & 0.7 \\
$a^{*}(\AA)$ & 4.4 & 7.4 & 5.2 & 10.4 \\
$\varrho_{\mathrm{M}}\left(10^{3} \mathrm{~kg} / \mathrm{m}^{3}\right)$ & 5.3 & 4.13 & 2.33 & 2.33 \\
$c_{1}\left(10^{3} \mathrm{~m} / \mathrm{s}\right)$ & 5.21 & 6.3 & 9.1 & 9.1 \\
$c_{\mathrm{t}}\left(10^{3} \mathrm{~m} / \mathrm{s}\right)$ & 3.0 & 3.54 & 4.55 & 4.55 \\
\hline
\end{tabular}

d) Ultrasonic relaxation attenuation in the case of $\mathrm{Si}(\mathrm{In}), \mathrm{Si}(\mathrm{B})$, and $\mathrm{GaAs}(\mathrm{Mn})$ can be explained by Orbach-like relaxation rates assuming a level at $4.2,2.0$, and $3.1 \mathrm{meV}$, respectively, above the relaxing ground state $[5,6]$. In the case of $\mathrm{Si}(\mathrm{B})$ the given value is less sure, because Raman and Orbach relaxations seem to be involved.

e) Raman scattering experiments in the case of $\mathrm{GaP}(\mathrm{Zn})$. They show some additional structure $2.7 \mathrm{meV}$ apart from the ground state [7].

A comparison of the resonance width and the temperature dependence of the whole scattering rate with the experimental data will be shown in a detailed discussion of a forthcoming paper.

In conclusion we state that the resonance structure, which has been seen by several types of experiments, can be accounted for by the Jahn-Teller effect of the $\Gamma_{8}$ ground state of deep effective mass acceptors.

\section{Conclusion}

To derive our results we started with Hamiltonian (1), which describes a dynamic Jahn-Teller system of $\Gamma_{8}-(t+e)$ type. In contrast to former calculations of phonon scattering with second-order Born approximation [10], we used a nonperturbative approach (Green's functions and transformation techniques) for a better inclusion of the internal phonon dynamics. Our formulas contain the perturbative results of [10] for small coupling parameters $D^{\varepsilon}$ and $D^{\tau}$ as a special case.

A better knowledge of the wave function of the deeper effective mass acceptors and a calculation of the quenching factors for the coupling constants (extending Morgan's equal-coupling case) are necessary for an absolute determination of the resonance.

\section{Acknowledgement}

We like to thank J. Maier for critical reading and suggestions to the paper. $\mathrm{He}$ also helped in preparing the manuscript.

\section{References}

[1] T. N. Morgan, Phys. Rev. Letters 24, 887 (1970).

[2] W. Korr, Solid State Phys. Б, 257 (1957).

[3] A. de Combaried and K. Lassmani, Phonon Scattering in Solids, Plenum Press, New York 1976 (p. 340). 
[4] H. Schenk, W. Forkel, and W. Eisenmenger, Frühjahrstagung DPG, Freudenstadt 1978.

[5] K. Lassucann and Hp. Schad, Solid State Commun. 18, 449 (1976).

[6] HP. SCHAD and K. Lassmann, Phys. Letters A56, 409 (1976).

[7] L. L. Chase, W. Hayes, and J. Ryan, J. Phys. C 10, 2957 (1977).

[8] R. SAUER, W. Schmid, and J. Weber, Solid State Commun. 27, 705 (1978).

[9] W. H. KLeINeR and L. M. Roth, Phys. Rev. Letters 2, 334 (1959).

[10] K. Suzuki and N. Mrkoshrba, Phys. Rev. B 3, 2550 (1971).

[11] W. Thonson and W. Morfit, Phys. Rev. 108, 1251 (1957).

[12] L. J. SchIFr, Quantum Mechanics, 3. ed., McGraw-Hill Publ. Co., New York 1968.

[13] H. Hasegawa, Phys. Rev, 118, 1513 (1960).

[14] M. V. KLeIN, Phys. Rev. B 186, 839 (1969).

[15] M. RUEFF, E. Sigmund, and M. WAGNer, phys. stat. sol. (b) 81, 511 (1977).

[16] E. Sigmond and M. Wagner, Z. Phys. 268, 245 (1974).

[17] M. ScHMrIDT, Thesis, Stuttgart 1975.

[18] H. Chandrasekhar, P. Frsher, A. Ramdas, and S. Rodrigues, Phys. Rev. B8, 3836 (1973). 